\title{
Analysis and Modeling of the Galvanic Skin Response Spontaneous Component in the context of Intelligent Biofeedback Systems Development
}

\author{
A. Unakafov \\ Southern Federal University, Taganrog Institute of Technology, Department of Computer Engineering; RITM OKB ZAO. \\ Voskov st.,102, 6, 347924, Taganrog, Russian Federation \\ E-mail: anton@rista.ru
}

The paper presents an approach to galvanic skin response (GSR) spontaneous component analysis and modeling. In the study a classification of biofeedback training methods is given, importance of intelligent methods development is shown. The INTENS method, which is perspective for intellectualization, is presented. An important problem of biofeedback training method intellectualization estimation of the GSR spontaneous component - is solved in the main part of the work. Its main characteristics are described; results of GSR spontaneous component modeling are shown. Results of small research of an optimum material for GSR probes are presented.

Keywords: galvanic skin response, spontaneous reactions, biofeedback, skin conductivity measurement, intelligent, modeling.

\section{INTRODUCTION}

$\mathrm{T}$ HE PROBLEM of the control and correction of the human functional state is of high urgency today. Increase of requirements to the human activity in interaction with the sophisticated equipment leads to the growth of nervousemotional load and formation of steady stressful statuses, development of neurosis and psychosomatic diseases. Elimination of such distresses purely pharmacological is often inaccessible because of contra-indications, acquired tolerance and by-effects, therefore psychophysiological methods of the person's emotional state correction widely develop. One of the largest groups of such methods is indirect biofeedback therapy (biofeedback training).

It is possible to divide methods of emotional state correction and, in particular - biofeedback training, into three groups: non-adaptive, adaptive and intelligent.

Non-adaptive methods. In such methods, there is no mechanism of procedure's correction depending on results of influence on the patient. The structure of non-adaptive method is presented in Fig.1, where TCA is Training Control Algorithm, the basis of the method.

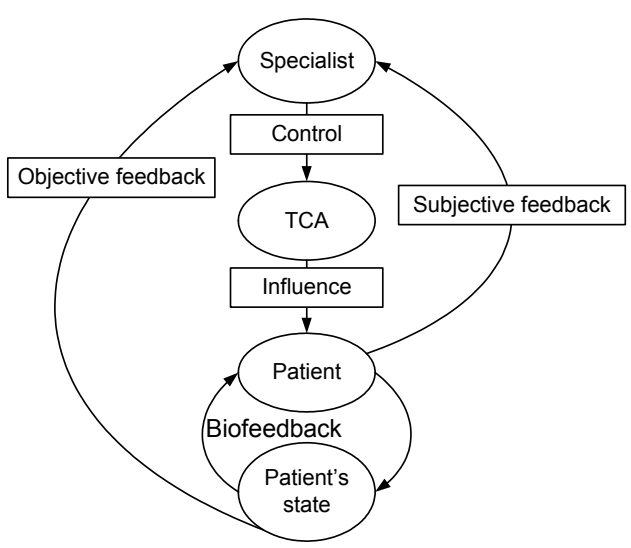

Fig.1 General view of the non-adaptive method
Influence adjustment is made by the expert (the psychologist, the psychophysiologist or the psychotherapist) based on an objective (the control of a patient state) and-or a subjective feedback (interrogation of the patient).

Common disadvantage of non-adaptive methods is their selective efficiency in case of simple methods, such as [1] and high load on the expert who is carrying out the procedure in case of sophisticated methods, such as [2].

Essential increase of biofeedback training efficiency is possible at the expense of approach personalization: maintenance of the maximum patient's sensitivity to perception of internal sensations [3], use of individually selected modes of the biofeedback training [4]

To solve these problems, adaptive methods are used (for example [5], [6]). They contain mechanisms of automatic adjustment of influence on the patient depending on his/her state. The structure of an adaptive method is presented in Fig.2.

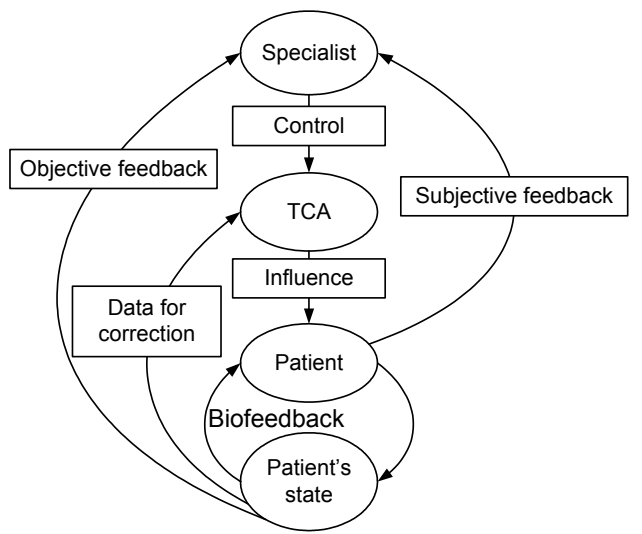

Fig.2 General view of the adaptive method

Though adaptive methods provide very high efficiency of training, in hard cases flexibility of the method appears 
insufficient and in such cases the result depends on experience and professional skills of the expert carrying out the procedure. Now the demand for highly skilled psychophysiologists grows and it is not fully satisfied.

To compensate for the lack of experts, to facilitate their work and raise its efficiency, intellectual methods can be used. Their structure is presented in Fig.3.

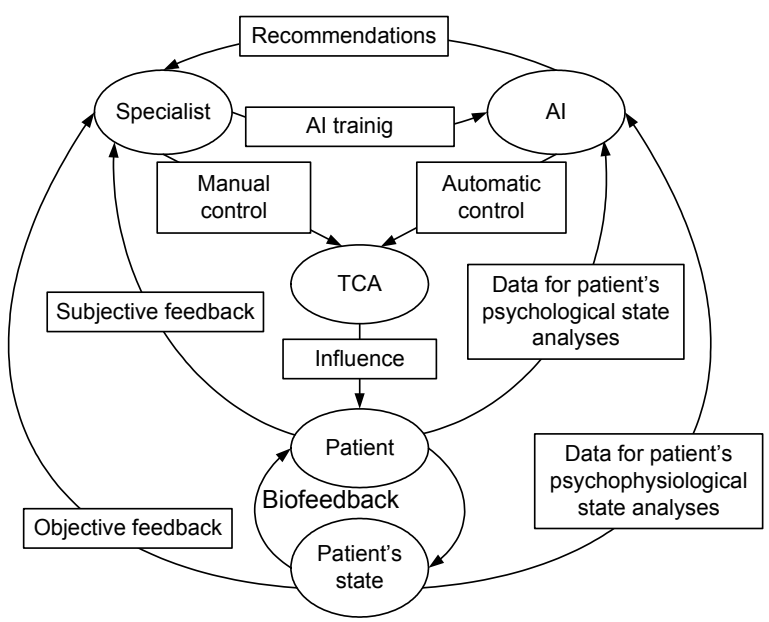

Fig.3 General view of the intelligent method

The intellectual method includes the mechanism of training conditions expert estimate (an artificial intellect, AI). It provides automatic development of influence tactics and, if necessary, delivery of recommendations to the expert who is carrying out the procedure. So, the training procedure can be carried out in two modes: manual (under control of the doctor) and automatic (under control of the AI). Thus, unlike the adaptive methods, which are only a convenient tool for the expert, intellectual methods can not only simplify his/her work, but also replace the expert.

Formulation and realization of intellectual methods is a current problem, which is poorly solved at present. There are some general ideas concerning formulation of intellectual methods in a number of sources (for example, [7]), however this problem is not fully solved yet.

Formulation of intellectual methods based on existing adaptive ones is obviously a promising area of research. For this purpose, it is necessary to develop the intellectual block (AI in Fig.3) providing patient's model construction based on the analysis of his/her physiological parameters and the psychological status.

One of the most useful and popular physiological parameters for biofeedback is electrodermal activity or Galvanic Skin Response (GSR) - the electric reaction measured from the surface of the skin and reflecting change of its bioelectric properties.

In this paper an intelligent hardware-software biofeedback system based on GSR is described. The biofeedback training method is considered in section 2.A, and its realization in section 2.B. The problem of the analysis and modeling of spontaneous reactions is set and solved in sections 2.D and 2.E.

For quality GSR analysis, quality measurement of GSR is required as a prerequisite. In section 2.C two types of GSRprobes are compared.

\section{Subject \& Methods}

\section{A. Biofeedback method description}

The proposed INTENS method is based on the adaptive method of biofeedback stress-resistance training INTEX [6], [8]. The course of training consists of four stages. Each of them consists of several sessions, every session takes about 10 minutes.

At the preliminary stage, features of spontaneous activity of the patient are studied. It is found out whether the patient can pass the training.

The aim of the first stage is to make the unconditioned orientated reflex to 2 new stimuli (high and low pitch sounds) fade away. The stage is considered to be completed, if at the beginning of a new session the psycho-emotional reaction to BOTH stimuli is rather weak.

At the second stage the conditioned defensive reflex to a high-pitch sound develops. The patient is exposed to these 2 stimuli at random, and the high pitch sound is accompanied by a discomfort electric stimulation. A conditioned reflex to a high pitch sound - danger - develops in the patient. This stage is considered to be completed, if at the beginning of a new session the psychoemotional reaction (without discomfort stimulation) to the high pitch sound considerably exceeds the reaction to the low pitch one. Then it is time to go to the third stage.

At the third stage, his/her reaction to the stimuli is shown to the patient. It is explained to the patient that if he suppresses his/her fear of the high pitch sound so that the reaction doesn't exceed, for example, $90 \%$ of that initial one, then there will be no discomfort electric stimulation. The patient deliberately and actively suppresses the autonomic component of the conditioned reflex due to relaxation and self-control. The session lasts until 8-10 dangerous stimuli are given. If his/her reaction to another high pitch signal does not exceed the specified threshold, the threshold for the next comparison is decreased. If the reaction does exceed the threshold, the discomfort stimulation is given automatically and the threshold is not changed. The stage is considered to be completed when there is no discomfort electric stimulation within the session, i.e. the threshold decreased at every step has never been exceeded.

How the amplitude of GSR changes in reply to stimuli is rather individual, before each session calibration - an estimation of reaction of an organism on normalized test influence (discomfort stimulation or a deep breath) is performed . Maximum GSR value during calibration is fixed and during a session is used for calculation of relative GSR values (in percentage of calibration GSR value).

For to automate the proposed method, it is necessary to 
develop the mechanism of the person's condition estimation enabling to determine whether the patient should go to the next stage of the biofeedback training. Thus, the following questions need to be answered:

1) Whether the patient can start training (transition from the preliminary to the first stage). Solution: analysis of the patient's psychophysiological condition, in particular spontaneous reactions, as the considerable quantity and high amplitude of spontaneous reactions, on the one hand, make the training impossible, and on the other hand - indicate the adverse psychological condition of the patient, which requires a special approach, for example - prescription of sedatives

2) Whether the orientated reflex has faded away (transition from the first to the second stage). Solution: check whether reactions to the stimuli exceed the maximum level of spontaneous reactions.

3) Whether the conditioned defensive reflex to a high-pitch sound has developed (transition from the second to the third stage). Solution: check whether the reaction to a high-pitch sound (without discomfort stimulation) exceeds the maximum level of spontaneous reactions appreciably (for example more than twice).

Solving all these problems requires the analysis of spontaneous GSRs, which are not studied enough as yet. When analyzing and modeling GSR, spontaneous activity is often neglected [9]. At the same time, it should be noted that values of spontaneous reaction can be above values of reaction to the stimuli. It can considerably complicate the analysis of the person's condition. Thereupon studying, analysis and modeling of spontaneous GSRs is important.

\section{B. Biofeedback system and GSR measuring}

The complex realizing the INTENS method consists of the following parts:

- PC program that provides session performance, signaling and display of the patient's reactions;

- measurement and stimulation device (on the basis of ATMega microcontroller), that transfers GSR measurements to the PC and provides the discomfort electric stimulation;

- GSR probes;

- stimulating electrode.

GSR probes are to be attached to the ring and index finger of one hand.

GSR measurement is carried out by direct current $2.5 \mu \mathrm{A}$. Value of electrode potential is measured and the skin conductance is calculated, phasic and tonic skin conductance are separated.

Further we will consider only phasic skin conductance (PSC, $S(t)$ ). It consists of:

- Skin Conductance Level (SCL) - PSC level corresponding to full calm, its changes are related with fluctuations of tonic skin conductance and if they are absent, SCL can be considered a constant. Further, we will consider $S(t)$ cleared from SCL;
- Skin Conductance Reaction (SCR, $\left.S_{R}(t)\right)$ - short, within several seconds, PSC changes in response to stimuli;

- Spontaneous Skin Conductance Reaction $\left(\mathrm{SSCR}, S_{S}(t)\right)$

- PSC changes not connected with any stimulus.

\section{Choose the Probe Material}

Research of an optimum material for GSR probes has been carried out. Plate probes of two types were investigated: stainless steel probes and brass probes, covered with silver.

5 people participated in the research that consisted of four stages, each one 10 minutes long. At $1^{\text {st }}$ and $2^{\text {nd }}$ stages the silver covered probes were placed on fingers of the left hand, steel probes - on the right; at $3^{\text {rd }}$ and $4^{\text {th }}$ stages probes interchanged the position. At $1^{\text {st }}$ and $3^{\text {rd }}$ stages PSC was measured in a quiescent state, at $2^{\text {nd }}$ and $4^{\text {th }}$ stages - with giving discomfort electric stimulation (up to 8 times during a stage) in casual moments. Time between research stages was not less than 20 minutes.

The conducted research has shown that results of PSC measurement almost do not depend on the hand on which the probes are set. At the same time, measurements were found to be reliably dependent on the material of probes. When silver covered probes were used, lower noise and higher sensitivity of PSC were observed. PSC changes in response to discomfort electric stimulation with silver covered probes were more than two times (on the average $-2.4 \pm 0.38$ ) higher than when steel probes were used.

\section{Analysis of patient's spontaneous activity}

We consider further that external stimuli do not influence the patient, so $S(t)=S_{S}(t)$.

As characteristics of spontaneous reactions, we propose to use the occurrence of spontaneous reactions $p_{s}$ and their typical (average) duration $T_{s}$, calculated by the following rules.

The beginning of $\mathrm{k}$-th spontaneous reaction was registered at the moment $t_{k}^{0}$, when $S(t)$ was increasing for $t \in\left[t_{k}^{0}, t_{k}^{0}+t_{i n c}\right]$, where $t_{\text {inc }}=0.3 \mathrm{sec}$, minimal time of $S(t)$ increase during spontaneous reaction.

The end of $\mathrm{k}$-th spontaneous reaction was registered at the moment $t_{k}^{1}$, when $S(t)>0$ for $t \in\left(t_{k}^{0}, t_{k}^{1}\right)$ and $S\left(t_{k}^{1}\right)=0$.

We designate the total quantity of spontaneous reactions as $N_{s}$. Average duration of spontaneous reactions $T_{s}$ and occurrence of spontaneous reactions $p_{s}$ can be calculated as follows: $T_{s}=\frac{1}{N_{s}} \sum_{k=1}^{N_{s}}\left(t_{k}^{1}-t_{k}^{0}\right), p_{s}=\frac{N_{S}}{T_{\text {Full }}-T_{S} N_{S}}$, where $T_{\text {Full }}$ - duration of recording.

The analysis of PSC records allows detecting four appreciably different states of $S_{S}(t)$ process:

- the quiescent state (“ 0 "): weak PSC deviations from 0 ;

- the activation state ("1"): appreciable increase of PSC; 
- the transitive state (“2”): weak PSC deviations from the maximum level;

- the relaxation state (" 3 "): appreciable decrease of PSC.

Such division corresponds to existing psychophysiological views [10]. It is clear that states " 1 ", " 2 " and " 3 " can occur only sequentially, one by one.

Thus, maximum durations of the states " 1 ", " 2 " and " 3 " ( $T_{1}, T_{2}$ and $T_{3}$ ), according to experimental data, are generally related as: $T_{1} \approx 2 T_{2} \approx \frac{2 T_{3}}{3}$, and the average duration of a state is two times less than maximal. Thus, as $T_{S}$ - average duration of spontaneous reactions, $T_{S}=\frac{T_{1}+T_{2}+T_{3}}{2}$ and it is simple to recover $T_{1}, T_{2}$ and $T_{3}: T_{1} \approx \frac{2}{3} T_{S}, T_{2} \approx \frac{1}{3} T_{S}$, $T_{3} \approx T_{S}$.

\section{E. Model of patients spontaneous activity}

The problem of spontaneous reactions $S_{S}(t)$ modeling can be divided into two sub-problems:

- modeling of probabilities of transition between the states.

- modeling of $S_{S}(t)$ behavior in each condition.

It should be noted that it is possible to consider probability of transition from quiescent state to activation state $p_{01}$ as a constant, but probabilities of transition between states " 1 " and " 2 " " 2 " and " 3 ", 3 " and " 0 " obviously depend on how long the process is in a current condition. So, $S_{S}(t)$ can be presented as the following process (Fig.4).

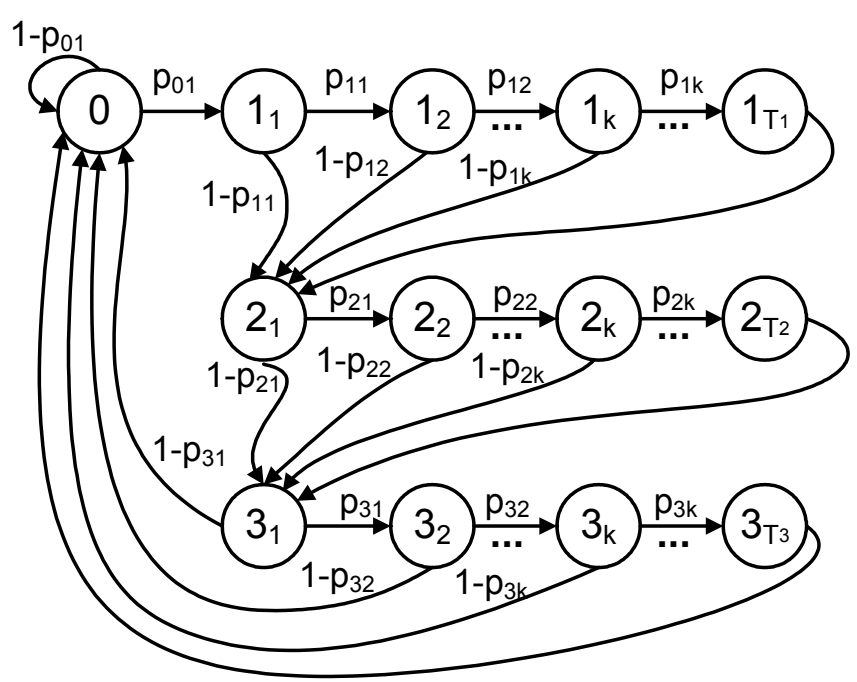

Fig.4 SSCR as a Markov process

According to this representation, the most natural is $S_{S}(t)$ modeling through Markov process. Its formal description is given below (modeled process will be denoted as $\tilde{S}_{S}(t)$ ).
As follows from Fig.4, the total number of process states $N$ is equal to $1+T_{1}+T_{2}+T_{3}$. As an initial state of system we always consider $« 0 »$, i.e. a quiescent state.

First, we consider probabilities of transition between conditions using the following denotations: for $i=\overline{1,2,3}, p_{i k}$ - probability of transition from the state " $i_{k}$ " to state " $i_{k+1}$ "; $p_{01}$ - probability of transition from the condition " 0 " to " $1_{1}$ ". It is obvious from Fig.4 that all nonzero probabilities can be denoted through these probabilities.

The probability of transition from quiescent to activation state $p_{01}$ is an important characteristic. The higher $p_{01}$ is, the higher the spontaneous reaction occurrence is, at $p_{01}=0$ there are no spontaneous reactions.

Below, requirements for transition rules within a state are formulated. Let us assume that $p_{s}$ and $T_{s}$ of the patient are known (received experimentally). Using them, one can calculate $T_{1}, T_{2}, T_{3}$ as well as $p_{01}: p_{01}=\frac{1}{n} p_{s}(n-$ quantity of measurements $S_{S}(t)$ per second and, accordingly, state change frequency, in our case $n=25$ ).

Experiments show that the probability $q_{i m}$ of process stay in the state $« i »$ during $m$ steps is distributed under the Gaussian law with population mean $\frac{T_{i}}{2}$ and mean square deviation $\frac{T_{i}}{6}$.

$q_{i m}=\frac{1}{\frac{T_{i}}{6} \sqrt{2 \pi}} \int_{m-\frac{1}{2}}^{m+\frac{1}{2}} e^{-\frac{1}{2}\left(\frac{x-\frac{T_{i}}{2}}{\frac{T_{i}}{6}}\right)^{2}} d x=\frac{6}{T_{i} \sqrt{2 \pi}} \int_{m-\frac{1}{2}}^{m+\frac{1}{2}} e^{-\frac{1}{2}\left(\frac{6 x}{T_{i}}-3\right)^{2}} d x$

Now we can evaluate $p_{i k}$.

$$
\begin{gathered}
q_{i m}=\left(1-p_{i m}\right) \prod_{k=1}^{m-1} p_{i k}=q_{i m-1} \frac{\left(1-p_{i m}\right)}{\left(1-p_{i m-1}\right)} p_{i m-1} \\
p_{i m}=1-\frac{1-p_{i m-1}}{p_{i m-1}} \frac{q_{i m}}{q_{i m-1}}=1-\frac{1-p_{i m-1}}{p_{i m-1}}\left(\int_{m+\frac{1}{2}}^{\int_{m-\frac{3}{2}}^{-\frac{1}{2}\left(\frac{6 x}{T_{i}}-3\right)^{2}} d x} \frac{\int^{m-\frac{1}{2}} e^{-\frac{1}{2}\left(\frac{6 x}{T_{i}}-3\right)^{2}} d x}{\int^{2}}\right)
\end{gathered}
$$

For a final specification of the recurrence formula for $p_{i m}$ it is necessary to determine $p_{i 1}$ (4).

$$
p_{i 1}=1-q_{i 1}=1-\frac{6}{T_{i} \sqrt{2 \pi}} \int_{\frac{1}{2}}^{\frac{3}{2}} e^{-\frac{1}{2}\left(\frac{6 x}{T_{i}}-3\right)^{2}} d x
$$


Probability $p_{3 k}$ must be set differently, otherwise the transition from state " $3_{k}$ " to state " 0 " is possible only when $\tilde{S}_{S}(t) \neq 0$, which is incorrect. To provide correct $\tilde{S}_{S}(t)$ behavior, one should not write down $p_{3 k}$ in an explicit form, but set a condition for the transition from " $3_{k}$ " to " 0 " in a form of inequation $\tilde{S}_{S}(t) \leq 0$.

We describe behavior of a process in each condition as a set $B=\left\{b_{i k}\right\}$, where $b_{i k}$ - increment $\tilde{S}_{S}(t)$ in a state " $i_{k}$ ".

In the quiescent state, weak deviations of $\tilde{S}_{S}(t)$ from 0 should take place, it is admissible to neglect them $b_{00}=0$ $\mu$ Siemens.

In the activation state, $\tilde{S}_{S}(t)$ increasing should be observed. From experimental data, one can consider that it is constant: $b_{1 k}(j)=6 \mu$ Siemens.

During the transition period, weak deviations of $\tilde{S}_{S}(t)$ from maximum should be observed: $b_{2 k}=\frac{\operatorname{sign}\left(\frac{T_{2}}{4}-k\right)}{2}$ $\mu$ Siemens, where function $\operatorname{sign}\left(\frac{T_{2}}{4}-k\right)=\left\{\begin{array}{l}-1, k<\frac{T_{2}}{4} \\ 1, k \geq \frac{T_{2}}{4}\end{array}\right.$ is used to provide in the transitive state an expressed maximum of $\tilde{S}_{S}(t)$, typical for real PSC records.

In the relaxation state, appreciable decrease of $\tilde{S}_{S}(t)$ should be observed. It is known that PSC "impulse" (which is response to a patient reaction), has a specific shape asymmetry: steep leading edge and more gentle falling edge [11]. It corresponds to experimental data according to which decrease of $S_{S}(t)$ in the relaxation state occurs more slowly than its increase in the activation state. Therefore, we can set $b_{3 k}=-4 \mu$ Siemens.

\section{RESULTS AND DISCUSSION}

Results of modeling are presented in Fig.5 and 6. Fig.5a, 6 a - real PSC of two patients without stimuli given - $S_{S}(t)$; $5 \mathrm{~b}, 6 \mathrm{~b}$ - results of the modeling, smoothed function $\tilde{S}_{S}(t)(5)$.

$$
\tilde{\tilde{S}}_{S}(t)=\frac{1}{3}\left(\tilde{S}_{S}(t-1)+\tilde{S}_{S}(t)+\tilde{S}_{S}(t+1)\right)
$$

Fig.5, 6 show that the method is rather effective (number and amplitude of spontaneous reactions are very similar for real and modeled PSC) and can be used for solving GSR modeling tasks. Modeling results can be considerably improved if Hidden Markov Model is used for modeling of the process $S_{S}(t)$. In this case, the behavior of process in each state $B=\left\{b_{i k}\right\}$ will be defined by stochastic function.

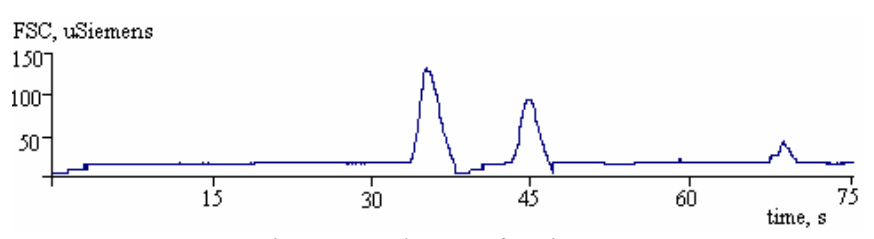

Fig.5a Real PSC of patient E

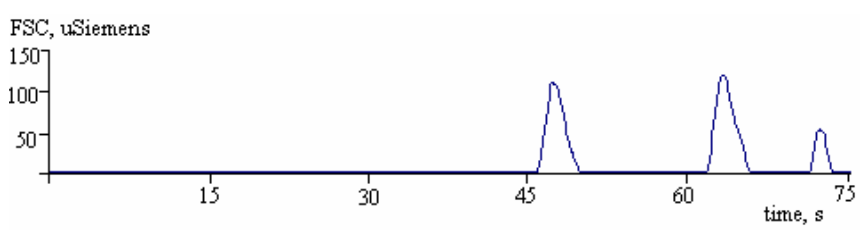

Fig.5b Modeled PSC of patient E

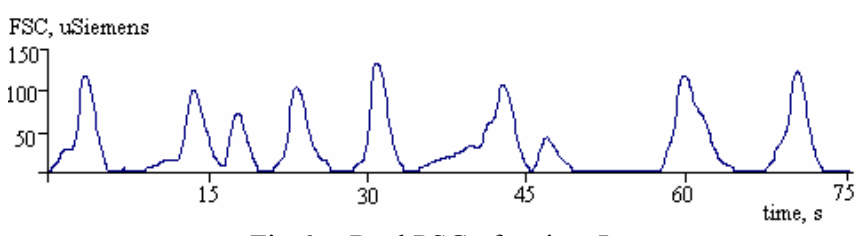

Fig.6a Real PSC of patient L

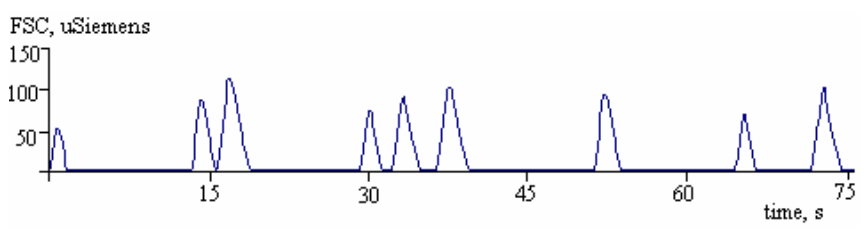

Fig.6b Modeled PSC of patient L

The results received in the present work allow to automate the process of biofeedback stress-resistance training by INTENS method.

It is promising to investigate the relationship between characteristics of spontaneous reactions used in this research and psychological condition of the patient. On one hand, it will raise the efficiency of modeling, and on the other hand it will allow using GSR analysis for diagnostic purposes and for monitoring the success of the course.

\section{REFERENCES}

[1] Wood, M., Forbes, A., Rhys, K. (2005). U.S. Patent No. 089856. Washington, D.C.: U.S. Patent and Trademark Office.

[2] Adamchuk, A.V., Zacharov, S.M., Skomorochov, A.A. (2002). Multifunctional multiparametrical rehabilitation complex for biomanagement. In Shtark B.M., Schwatz M. (eds.) Biofeedback 4: Theory and Practice. Novosibirsk, Russia: CERIS, 287-291.

[3] Lehrer, P.M. (1998). Emotionally triggered asthma: a review of research literature and some hypothesis for self-regulation therapies. Applied Psychophysiology and Biofeedback, 32 (4), 13-41. 
[4] Thatcher, R.W. (2000). EEG operant conditioning (biofeedback) for traumatic brain injury. Clinical Electroencephalography, 31 (1), 38-44.

[5] Suvorov, N.B. (2006). Psychophysiological Training of operators in adaptive biofeedback cardiorhythm control. The Spanish Journal of Psychology, 9 (2), 193-200.

[6] Unakafov, M.A. (2005). Russia Federation Patent No. 2251972. Moscow: Russian Federal Service for Intellectual Property, Patents and Trademarks.

[7] Bersak, D., McDarby, G., Augenblick, N., McDarby, P., McDonnell, D., McDonald, B., Karkun, R. (2001, September) Intelligent Biofeedback using an Immersive Competitive Environment. Retrieved December 20, 2008 from http://medialabeurope.org/mindgames/publications/publicationsAtlanta2001rev3.pdf
[8] Unakafov, A.M. (2008). Adaptive method of hardwaresoftware support of person's emotional self-checking training processes. Izvestiya TTI SFU, 6, 37- 41.

[9] Fernandez, R., Picard, R.W. (1998). Signal processing for recognition of human frustration. In Proceedings of the 1998 IEEE International Conference on Acoustics, Speech and Signal Processing, 12-15 May 1998. Seattle, USA, 3773 - 3776.

[10] Suhodoev, V.V. (1997). Estimation of the person psychophysiological status activation components on galvanic skin response. Psychological Journal, 18 (5), 305-328.

[11] Cacioppo, J.T., Tassinary, L.J. (eds.) (1990) Principles of Psychophysiology: Physical, Social, And Inferential Elements. Cambridge University Press. 